\title{
An Augmented Reality Navigation System for Computer Assisted Arthroscopic Surgery of the Knee
}

\author{
Oliver Tonet ${ }^{1}$, Giuseppe Megali ${ }^{1}$, Simona D’Attanasio ${ }^{1}$, Paolo Dario ${ }^{1}$, \\ Maria Chiara Carrozza ${ }^{1}$, Maurilio Marcacci ${ }^{2}$, Sandra Martelli ${ }^{2}$, \\ Pier Francesco La Palombara ${ }^{2}$ \\ ${ }^{1}$ MiTech Lab, Scuola Superiore S. Anna, Via Carducci 40, 56127 Pisa, Italy \\ oly@sssup.it, peppe@mail-arts.sssup.it, dario@arts.sssup.it, \\ chiara@arts.sssup.it \\ http://www-mitech.sssup.it/ \\ ${ }^{2}$ Biomechanics Lab, Istituti Ortopedici Rizzoli, Bologna, Italy \\ \{M.Marcacci, S.Martelli, F.La.Palombara\}@biomec.ior.it \\ http://www.ior.it/biomec/
}

\begin{abstract}
This work presents a prototype navigation system for computer assisted arthroscopic surgery. Arthroscopic surgery is minimally invasive and ensures fast post-surgical recovery. It requires, however, considerable dexterity due to the limited $2 \mathrm{D}$ view of the scene and cramped operating area. Augmented reality can help the surgeon overcome these limitations. The key feature of the system presented is an interactive graphical interface. A 3D model and two 2D projections of the patient's joint and models of the arthroscope and other sensorized surgical instruments are shown. The positions of all sensorized objects, including the patient's anatomy, are tracked in real-time by an optoelectronic localizer and reproduced in the virtual scene. The field of view of the arthroscope is dynamically highlighted on the 3D model of the joint, which is reconstructed from a preoperative CT/MRI data set and matched to the actual anatomy by means of an ICP-based non-fiducial registration algorithm. The prototype system has been positively evaluated by a selected group of skilled orthopaedic surgeons. Preliminary tests on the overall accuracy of the system show that errors can be kept within $3^{\circ} / 3 \mathrm{~mm}$.
\end{abstract}

\section{Introduction}

Arthroscopic surgery is widely used as a routine treatment for several joint disorders. Being minimally invasive, it ensures little tissue damage and faster post-surgical recovery. Arthroscopic surgical procedures require, however, considerable dexterity and skill due to the limited $2 \mathrm{D}$ view of the scene and the cramped operating area. Augmented reality systems enhance the surgical skills and help the surgeon overcome these limitations [1]. A navigation system, aimed at the improvement of the current state of the art of arthroscopic surgery has been developed and tested in vitro. The system requirements have been defined in collaboration with a team of experts in the field of arthroscopy. 


\section{Clinical Relevance}

Despite the strong social and economic importance that minimally invasive surgical techniques have achieved, still many technical problems are to be solved in order to improve the quality of the care delivered to patients. In arthroscopic surgery the narrow access imposes severe constraints on the operating workspace. Vision is limited by the small monoscopic field of view of the arthroscope and by image quality. Other sensory signals, such as tactile sense and force feedback are significantly reduced. Research efforts are currently devoted not only to restore, but also to enhance the surgeon's perceptive capabilities. The proposed augmented reality navigation system can provide guidance during the surgical procedure by integrating the images from the arthroscopic camera and other sensory data with a virtual environment.

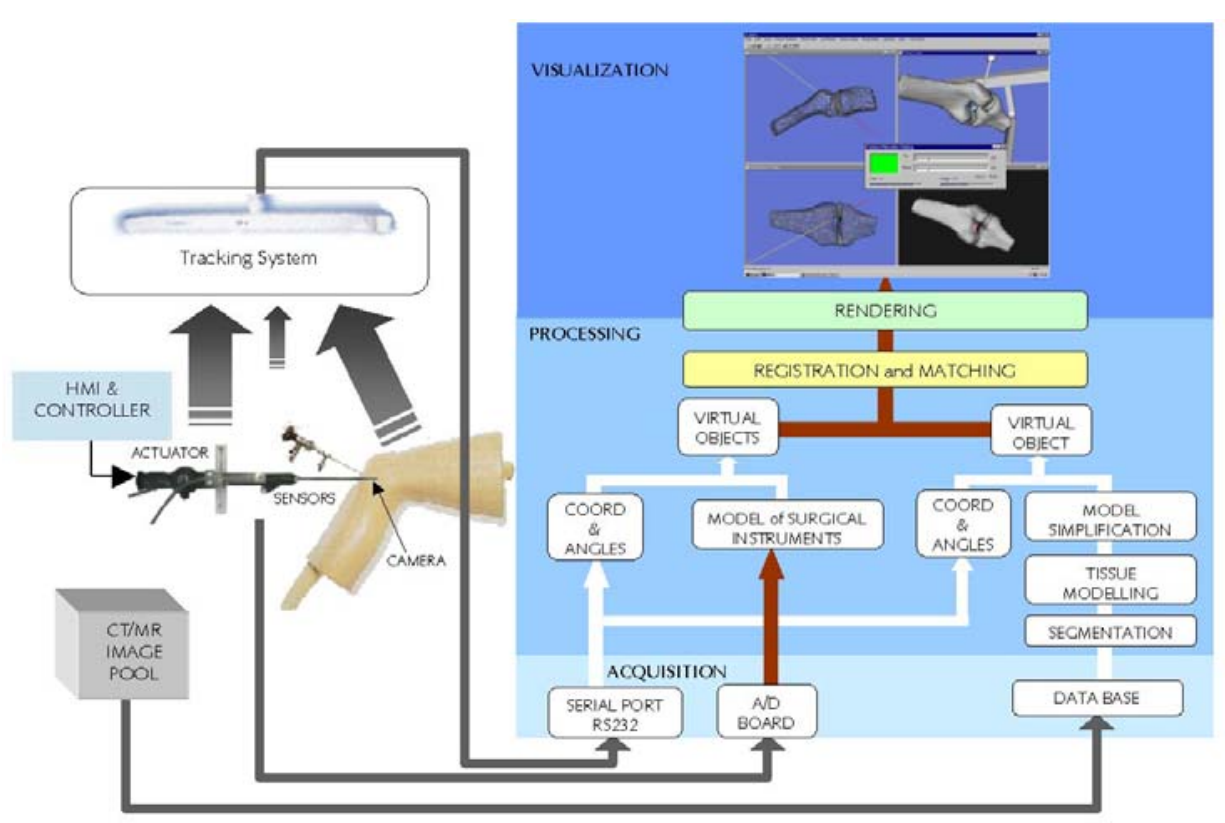

Fig. 1. A general scheme of the navigation system

\section{System Overview}

The system includes different intercommunicating modules. Some of them account for preoperative image processing, such as image processing modules for:

1. Segmentation of preoperative CT or MR images;

2. 3D reconstruction of the virtual model of the anatomy. 
Other modules are used intraoperatively, such as:

1. The registration module for matching preoperative data, consisting in a virtual model of the anatomical structures, and intraoperative data, consisting in the patient's anatomy;

2. A tracking module for the real time monitoring of the position of the surgical tools and anatomical structures;

3. A graphical human-machine interface displaying the enhanced reality scene.

To determine the position and orientation of the objects, the system uses a commercial optical localizer which employs three cameras located on a stand placed over the surgical scene and defines the coordinate system of reference of the operating room. The cameras detect IR pulses emitted by LED's placed on rigid frames fixed to the objects to track. The control unit of the localization system computes the coordinates of the LED positions by means of geometrical triangulation and determines the precise position and orientation of the objects present in the scene. The main function of the system is to help the surgeon drive the tools along a precise trajectory and maintain the desired tool positions and orientations with respect to the anatomical structures.

An accurate registration between the joint model and the patient's anatomy is essential to blend pre- and intraoperative data into integrated graphical objects. The implemented registration technique is the "constrained ICP", a modified version of the renowned Iterative Closest Point (ICP) algorithm [2]. The algorithm matches two different point sets belonging to the same object. In the proposed approach the first set is composed by points sampled from the preoperative 3D model, while the second set contains points collected intraoperatively with the optical digitiser. The first point set includes points from the areas which are actually reachable with the digitiser tip during the intervention. The innovation in this approach is to partition such point set into different subsets, each corresponding to a single detached area, and cluster the second data set in paired subsets. The registration algorithm has been designed so that the points included in each subset of the first data set are constrained to match their closest points in the corresponding subset of the second data set. This ensures a quicker and safer convergence towards an acceptable solution and removes the need for an initial guess assessment step. By integrating preoperative and intraoperative data, the system allows for virtual navigation within the joint during the planning phase, and computer guidance by augmented reality during the intervention.

The virtual scene of the operating room is shown on a computer display, which contains four windows. The frame is structured as follows: the external viewpoint window displays the surgical scene as viewed from an external viewpoint. This view may be adjusted according to surgeon preference by changing camera position, orientation and magnification. For external reference, the main objects in the operating room are displayed together with the anatomical structures and the surgical tools. The lateral projection and the frontal projection windows show a translucent projection of the anatomical segments and lines representing the axes of the surgical tools for trajectory control. The arthroscope tracking light window shows the virtual model and highlights the anatomical area included in the arthroscope field of view.

A mechatronic arthroscope has been designed, manufactured in a prototype version and successfully integrated with the navigation system. The mechatronic arthroscope has a cable-actuated servomotor-driven multi-joint mechanical structure. It is 
equipped with a position sensor, measuring the orientation of the tip, and a force sensor, detecting possible contact with delicate tissues in the knee. Moreover it incorporates an embedded microcontroller for sensor signal processing, motor driving and interfacing with the surgeon and/or the system control unit [3].

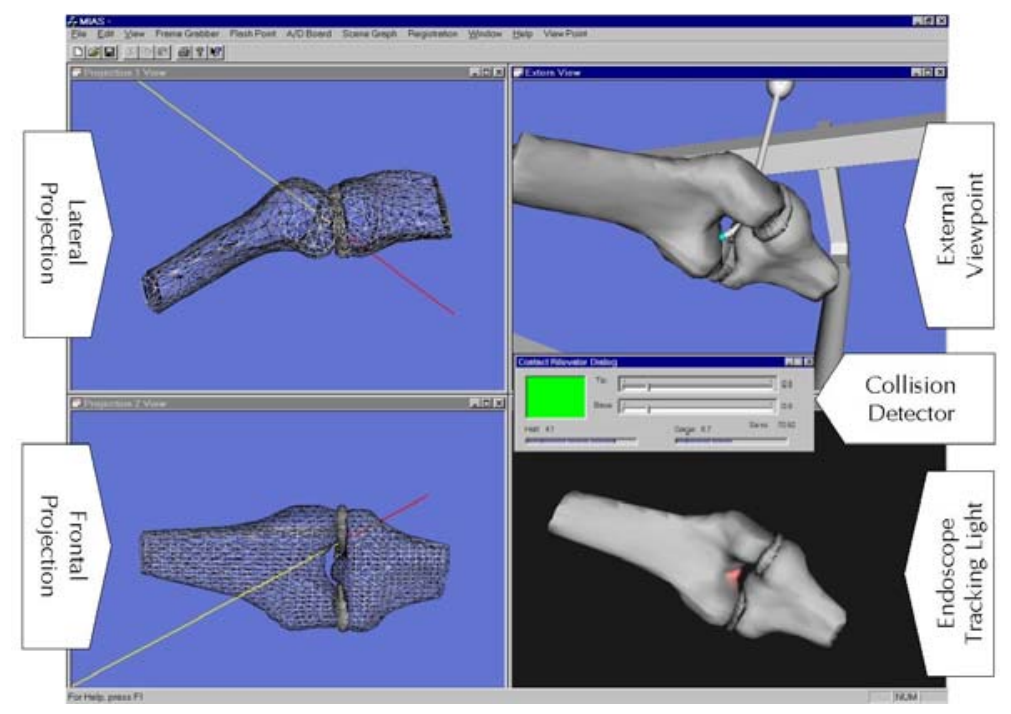

Fig. 2. The graphical interface of the navigation system

\section{$4 \quad$ Tests and Results}

The system has been tested using an artificial model of the human knee (Sawbones Europe $\mathrm{AB}$ ) including femur, tibia, patella, menisci, ligaments and the surrounding soft tissues. A virtual model of the knee was obtained from a CT scan of this model, after segmentation and $3 \mathrm{D}$ reconstruction. The points of the anatomy database were selected on the virtual model and registered with the reference frame of the $3 \mathrm{D}$ localizer (FlashPoint 5000 system, Image Guided Technologies Inc.). The position of three cameras, located on a frame placed over the surgical scene, defines the coordinates of the common reference frame. The cameras detect the IR pulses emitted by LED's mounted on the tools to be tracked. The LED position is reconstructed by means of geometrical triangulation. In the experiments, a square frame was used as support for four IR LED's. The frame was mounted on the external housing of a commercial $30^{\circ}$ arthroscope (Karl Storz Endoskope GmbH). The central control unit is an Intergraph TDZ 2000 GX1 workstation (Pentium II Xeon $450 \mathrm{MHz}, 128 \mathrm{MB}$ RAM, Windows NT 4.0) with RealiZm II 3D graphics board. Communication between the localizer and the workstation is based on a standard serial port. The navigation system has been developed in C++ language for Windows NT 4.0 
platform, using Microsoft Foundation Classes for the graphical user interface (GUI) and OpenGL Optimizer for 3D visualization.

The following table summarizes the system performance parameters as resulting from the laboratory trials.

Table 1. System performance

\begin{tabular}{lc}
\hline Overall System Error $^{1}:$ & $2.3 \mathrm{~mm}$ \\
\hline Acquisition error & $1.0 \mathrm{~mm}$ \\
Simplification error & $0.4 \mathrm{~mm}$ \\
Localization error & $0.5 \mathrm{~mm}$ \\
Registration error & $2.0 \mathrm{~mm}$ \\
\hline System Refresh Rate ${ }^{2}:$ & $13 \mathrm{~Hz}$ \\
\hline Localizer refresh rate $_{\text {GUI refresh rate }}$ & $14 \mathrm{~Hz}$ \\
\hline
\end{tabular}

\section{Conclusions}

Although further testing is essential to validate the system, the first trials have given positive feedback. The prototype system has been favorably evaluated by a selected group of skilled orthopedic surgeons. The use of general-purpose methods and the modularity of the architecture make the navigation system application-independent. It can be adapted to a variety of surgical procedures, including those featuring different graphical user interfaces, and can be used with both ordinary surgical tools and custom instrumentation.

\section{References}

1. Satava, R.M., Jones, S.B.: Current and Future Applications of Virtual Reality for Medicine. Proceedings of the IEEE 86(3) (1998) 484-489

2. Besl, P.J., McKay, N.D.: A Method for Registration of 3-D Shapes. IEEE Trans PAMI 14(2) (1992) 239-256

3. Dario, P., Carrozza, M.C., Marcacci, M., D’Attanasio, S., Magnani, B., Tonet, O., Megali, G.: A Novel Mechatronic Tool for Computer-Assisted Arthroscopy. IEEE Trans ITB 4(1) (2000) 15-29

\footnotetext{
${ }^{1}$ Mean difference between coordinates in the virtual scene and in the real environment.

${ }^{2}$ Inverse of the time needed by the system to acquire all data and update the virtual scene accordingly.
} 